\title{
Chinese Herbal Formula, Modified Danggui Buxue Tang, Attenuates Apoptosis of Hematopoietic Stem Cells in Immune- Mediated Aplastic Anemia Mouse Model
}

\author{
Jingwei Zhou, ${ }^{1,2}$ Xue Li, ${ }^{1}$ Peiying Deng, ${ }^{1}$ Yi Wei, ${ }^{1}$ Juan Liu, ${ }^{1}$ Meng Chen, ${ }^{3}$ Yamei Xu, \\ Dongmei Zhang, ${ }^{1}$ Lingqun Zhu, ${ }^{1}$ Lixia Lou, ${ }^{1}$ Bin Dong, ${ }^{1}$ Qiushuo Jin, ${ }^{1}$ and Limin Chai ${ }^{1}$ \\ ${ }^{1}$ Key Laboratory of Chinese Internal Medicine of Ministry of Education and Beijing, Dongzhimen Hospital, Beijing University of \\ Chinese Medicine, Beijing 100700, China \\ ${ }^{2}$ Department of Rheumatology, Dongzhimen Hospital, Beijing University of Chinese Medicine, Beijing 100700, China \\ ${ }^{3}$ School of Preclinical Medicine, Beijing University of Chinese Medicine, Beijing 100029, China \\ ${ }^{4}$ Department of Hematology \& Oncology, Dongzhimen Hospital, Beijing University of Chinese Medicine, Beijing 100700, China
}

Correspondence should be addressed to Limin Chai; liminchai@hotmail.com

Received 9 January 2017; Revised 1 June 2017; Accepted 12 July 2017; Published 16 August 2017

Academic Editor: Qingdong Guan

Copyright (C) 2017 Jingwei Zhou et al. This is an open access article distributed under the Creative Commons Attribution License, which permits unrestricted use, distribution, and reproduction in any medium, provided the original work is properly cited.

A derivative formula, DGBX, which is composed of three herbs (Radix astragali, Radix Angelicae sinensis, and Coptis chinensis Franch), is derived from a famous Chinese herbal formula, Danggui Buxue Tang (DBT) (Radix astragali and Radix Angelicae sinensis). We aimed to investigate the effects of DGBX on the regulation of the balance between proliferation and apoptosis of hematopoietic stem cells (HSCs) due to the aberrant immune response in a mouse model of aplastic anemia (AA). Cyclosporine (CsA), an immunosuppressor, was used as the positive control. Our results indicated that DGBX could downregulate the production of IFN $\gamma$ in bone marrow cells by interfering with the binding between SLAM and SAP and the expressions of Fyn and T-bet. This herbal formula can also inhibit the activation of Fas-mediated apoptosis, interferon regulatory factor-1-induced JAK/Stat, and eukaryotic initiation factor 2 signaling pathways and thereby induce proliferation and attenuate apoptosis of HSCs. In conclusion, DGBX can relieve the immune-mediated destruction of HSCs, repair hematopoietic failure, and recover the hematopoietic function of HSCs in hematogenesis. Therefore, DGBX can be used in traditional medicine against AA as a complementary and alternative immunosuppressive therapeutic formula.

\section{Introduction}

Aplastic anemia (AA) is a rare acquired bone marrow failure syndrome resulting from immune-mediated destruction of hematopoietic stem cells (HSCs), caused largely by many quantitative and qualitative defects in HSCs [1]. Allogeneic stem cell transplant can cure severe AA. Nevertheless, transplantation in patients who are old or lack family donors has many challenges [2]. The molecular basis of the aberrant immune response and deficiencies in HSC is now being defined genetically. Immunosuppression by antithymocyte globulins and cyclosporine A (CsA) is effective on restoring blood cell production in the majority of AA patients.
However, the incidence of relapse and evolution of clonal hematological diseases remain high [3].

Danggui Buxue Tang (DBT), one of the simplest traditional Chinese medicine (TCM) decoctions, has been used in the treatment of blood deficiency syndrome for more than 800 years in China. DBT is composed of two herbs, Radix astragali (Milkvetch Root) and Radix Angelicae sinensis (Chinese Angelica), boiled together at a 5:1 ratio. Pharmacological studies indicated that DBT has effects on bone development [4], blood enhancement [5], and immune stimulation [6]. Several studies have verified the hematopoietic function of DBT in a mouse model of bone marrow suppression. It can increase the production of peripheral blood and nucleated 


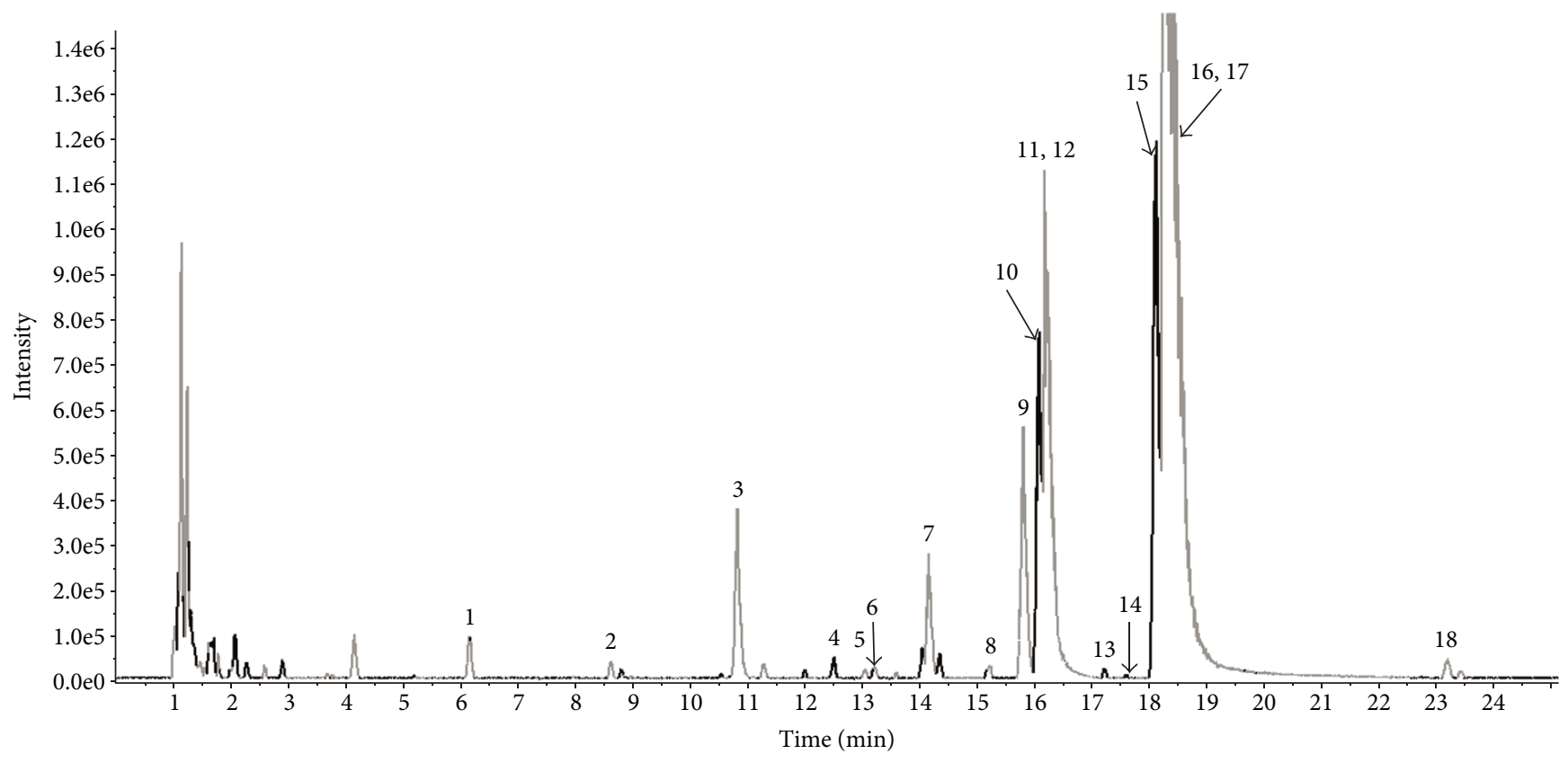

FIGURE 1: High-performance liquid chromatography-electrospray ionization/mass spectrometry ion chromatograms of lyophilized boiled aqueous extract of DGBX. Abscissa represents retention time, and ordinate represents chromatographic peak intensity.

bone marrow cells (BMCs) and the colony count of hematopoietic stem/progenitor cells (HSPCs) in vitro; regulate the expression of cytokines, such as erythropoietin, thrombopoietin, and granulocyte-macrophage colony-stimulating factor in bone marrow microenvironment; and contribute to cell cycle of HSPCs [7]. For the bone marrow failure syndrome resulting from immune-mediated destruction of HSC, we modified DBT to prepare a derivative herb formula (DGBX) by adding Coptis chinensis Franch (Coptidis rhizoma) for reinforcing the regulatory effect on aberrant immune response in the bone marrow of patients with AA. Pharmacological studies validated that Coptis chinensis Franch modulates immune responses and controls certain inflammation-related diseases [8]. The combination of these three herbs can contribute to multitargeting, multichannel immunosuppressive effects in AA treatment.

We investigated the possible mechanisms underlying the immunosuppressive and hematopoietic functions of DGBX in an immune-mediated AA mouse model. CsA was used as a positive control. The aim of this study was to identify the specific cellular targets involved in the immunosuppressive and hematopoietic functions of DGBX in AA treatment.

\section{Materials and Methods}

2.1. Preparation of Herbal Composition of DGBX. Radix astragali, Radix Angelicae sinensis, and Coptis chinensis Franch (total weight, $126 \mathrm{~g}$; individual ratio, 5:1:1) were boiled together in $6 \times$ volume of water for $0.5 \mathrm{~h}$. Then, residue from the first extraction was boiled in $8 \times$ volume of water for $25 \mathrm{~min}$. Finally, the filtered solutions were combined and concentrated into aqueous extracts containing $0.9 \mathrm{~g} / \mathrm{mL}$ of raw herbs.
2.2. High-Performance Liquid Chromatography-Electrospray Ionization/Mass Spectrometry. The herbal extracts were filtered using a $150 \mu \mathrm{m}$ standard test sieve and maintained in desiccators at $4^{\circ} \mathrm{C}$ until use. Lyophilized flower $(0.02 \mathrm{~g})$ was extracted with $5 \mathrm{~mL}$ of methanol/water $(v / v=1: 1)$ in the sonicator for $20 \mathrm{~min}$ at room temperature. The extract was filtered through a $0.22 \mu \mathrm{m}$ membrane. A $10 \mu \mathrm{L}$ aliquot of the extract was injected into the analytical column for analysis. The compounds were separated on a Phenomenex Kinetex $\mathrm{C} 18(2.6 \mu \mathrm{m}, 100 \times 2.1 \mathrm{~mm})$ operated at $35^{\circ} \mathrm{C}$. The mobile phase $(0.1 \%$ formic acid in water (A) and acetonitrile (B) as mobile phase) was delivered at a flow rate of $0.4 \mathrm{~mL} / \mathrm{min}$ under a gradient program. The diode-array detector was set at $254 \mathrm{~nm}$, and the online UV spectra were recorded in the scanning range of $190-400 \mathrm{~nm}$.

The optimized parameters for negative and positive modes were as follows: the ion spray voltage was set at 5500 (positive ion mode) and $-4500 \mathrm{~V}$ (negative ion mode); the turbo $\mathrm{V}$ spray temperature at $600^{\circ} \mathrm{C}$, nebulizer gas (gas 1) at 50 psi, heater gas (gas 2) at 60 psi, collision gas at medium, the curtain gas at $30 \mathrm{psi}$, and the declustering potential at 80 (positive ion mode) and $-80 \mathrm{~V}$ (negative ion mode). The collision energy was set at 35 (positive ion mode) and $-35 \mathrm{~V}$ (negative ion mode), and the collision energy spread was set at $15 \mathrm{~V}$ for MS/MS experiments. The data was acquired with IDA (information-dependent acquisition) method and analyzed by Peak View Software ${ }^{\mathrm{TM}} 2.2$ (SCIEX, Foster City, CA, USA).

2.3. Mice. BALB/c female mice ( $n=6$ per group, $7-8$ weeks old) and DBA/2 female mice ( 7 to 8 weeks old) were purchased from HFK Bioscience Co. Ltd. (Beijing, China). Animal care and use were in accordance with the institutional guidelines. All animal experiments were approved by the Institutional 
TABLE 1: Chemical components identified from DGBX by high-performance liquid chromatography-electrospray ionization/mass spectrometry.

\begin{tabular}{lccc}
\hline Peak & $t_{\mathrm{R}}(\mathrm{min})$ & $\mathrm{Formula}_{12}$ & Identification \\
\hline 1 & 6.14 & $\mathrm{C}_{11} \mathrm{H}_{12} \mathrm{~N}_{2} \mathrm{O}_{2}$ & L-tryptophan \\
2 & 8.61 & $\mathrm{C}_{17} \mathrm{H}_{20} \mathrm{O}_{9}$ & 3-O-Feruloylquinic \\
3 & 10.82 & $\mathrm{C}_{20} \mathrm{H}_{24} \mathrm{NO}_{4}$ & Magnoflorine \\
4 & 12.5 & $\mathrm{C}_{22} \mathrm{H}_{22} \mathrm{NO}_{10}$ & Calycosin-7-O- $\beta$-D-glucoside \\
5 & 13.04 & $\mathrm{C}_{10} \mathrm{H}_{10} \mathrm{O}_{4}$ & Ferulic acid \\
6 & 13.2 & $\mathrm{C}_{12} \mathrm{H}_{16} \mathrm{O}_{4}$ & Senkyunolide I \\
7 & 14.17 & $\mathrm{C}_{22} \mathrm{H}_{24} \mathrm{NO}_{4}^{+} \mathrm{Cl}^{-}$ & Tetradehydroscoulerine \\
8 & 15.22 & $\mathrm{C}_{22} \mathrm{H}_{22} \mathrm{NO}_{10}$ & Jatrorrhizine \\
9 & 15.81 & $\mathrm{C}_{20} \mathrm{H}_{20} \mathrm{NO}_{4}$ & Ononin \\
10 & 16.01 & $\mathrm{C}_{22} \mathrm{H}_{22} \mathrm{O}_{9}$ & Epiberberine \\
11 & 16.08 & $\mathrm{C}_{20} \mathrm{H}_{18} \mathrm{NO}_{4}$ & Coptisine \\
12 & 16.21 & $\mathrm{C}_{19} \mathrm{H}_{14} \mathrm{NO}_{4}$ & Calycosin-7-O- $\beta$-D-glucoside- ${ }^{\prime \prime}$-O-malonate \\
13 & 16.67 & $\mathrm{C}_{24} \mathrm{H}_{26} \mathrm{O}_{10}$ & Z-Butylidenephthalide \\
14 & 17.6 & $\mathrm{C}_{12} \mathrm{H}_{12} \mathrm{O}_{2}$ & Palmatine \\
15 & 18.12 & $\mathrm{C}_{21} \mathrm{H}_{25} \mathrm{NO}_{4}$ & Berberine \\
16 & 18.31 & $\mathrm{C}_{20} \mathrm{H}_{18} \mathrm{NO}_{4}$ & Calycosin \\
17 & 18.47 & $\mathrm{C}_{16} \mathrm{H}_{12} \mathrm{O}_{5}$ & Formononetin \\
18 & 23.19 & $\mathrm{C}_{16} \mathrm{H}_{12} \mathrm{O}_{4}$ &
\end{tabular}

Animal Care and Use Committee of the National Institute of State Scientific and Technological Commission.

2.4. Induction $A A$. BALB/c mice, except mice in the normal group, received a sublethal total body irradiation dose of 3.5 Gy from Model $143{ }^{137}$ Cesium $\gamma$-irradiator one hour before lymph node cell infusion. Inguinal, brachial, and axillary infusion of lymph node cells were obtained from female $\mathrm{DBA} / 2$ mice. Lymph cells $\left(1 \times 10^{6}\right)$ were injected into BALB/ c mice through the tail vein for inducing AA [9].

2.5. Drug Treatment. The treatment was initiated after lymph node cell infusion and lasted for 28 days. Mice were randomly divided into four groups: normal group, mice were fed the control diet and orally administered sterile saline; model group, mice were fed the same as the normal group; cyclosporine (CsA) group, CsA (batch number H10960122, Zhongmei Huadong Pharmaceutical Co. Ltd., Hangzhou, China) mice were fed the same control diet and orally daily administered $25 \mathrm{mg} / \mathrm{kg}$ CsA daily for 28 days; and DGBX group, mice were fed the same control diet and orally administered $6.3 \mathrm{~g} / \mathrm{kg}$ DGBX daily for 28 days. The mice were sacrificed on the 29th day after treatment. Mice were anesthetized by isoflurane anesthesia (2-3\% isoflurane with oxygen supply). Peripheral blood samples were collected by removing eyeballs, and BMCs were obtained by femoral cavity flushing.

2.6. Enzyme-Linked Immunosorbent Assay. Twenty-four hours after the last administration, $0.8 \mathrm{~mL}$ of peripheral blood was collected from each mouse by eyeball extirpation. Sera were isolated by centrifuging at $3000 \mathrm{rpm}$ and $4^{\circ} \mathrm{C}$ for $10 \mathrm{~min}$. The concentrations of interferon $\gamma(\operatorname{IFN} \gamma)$, interleukin-2 (IL-2), and tumor necrosis factor $\alpha$ (TNF $\alpha)$ were measured using ELISA kits (eBioscience, San Diego, CA, USA), according to the instructions provided by the manufacturer.

2.7. Fluorescence-Activated Cell Sorter Analysis. BMCs were obtained by femoral cavity flushing and filtered through a 200 -eye cell sieve mesh to obtain a single-cell suspension. To quantify the percentage of HSCs, CD117- and sac-1positive cells were washed and stained with anti-mouse CD117 (c-Kit) FITC and anti-mouse Ly-6A/E (Sca-1) PE antibodies (eBioscience). For the detection of apoptosis in BMCs, an Annexin V-FITC Apoptosis Detection Kit (eBioscience) was used. BMCs were stained with FITCconjugated annexin $\mathrm{V}$ and propidium iodide (PI). Flow cytometry was performed using a fluorescence-activated cell sorter Calibur cytometer and analyzed with CellQuest software (Beckman Coulter, Brea, CA, USA).

2.8. Immunofluorescence. To examine the expression and distribution of T-bet in BMCs, cells were fixed, permeabilized, and incubated with anti-human/mouse T-bet PE $(1: 100)$ (eBioscience). To identify changes in binding of signaling lymphocyte activation molecule (SLAM) and SLAMassociated protein (SAP), cells were stained with antimouse CD150/SLAM PE antibody (1:50) (eBioscience). After permeabilization, cells were incubated with SH2D1A/ SAP antibody (FITC) $(1: 100)$ (EterLife, Birmingham, UK). Confocal fluorescence microscopy images were captured using a Leitz/Leica TCSSP2 microscope (Leica Lasertechnik $\mathrm{GmbH}$, Heidelberg, Germany). The fluorescence intensity was quantified with ImageJ. At least 50 cells from 3 different areas of each chamber were measured. 


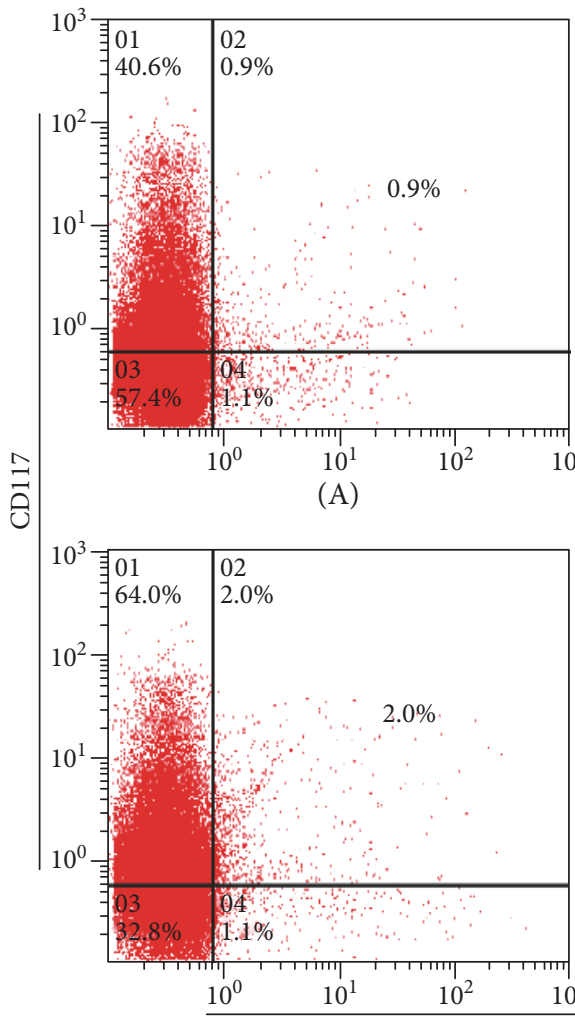

(C)

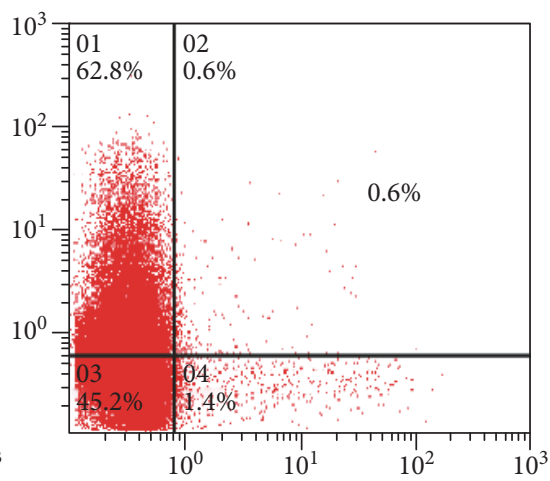

(B)

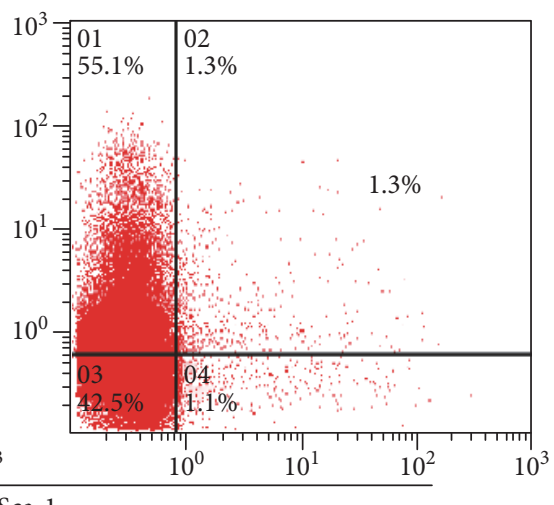

(D)

(a)

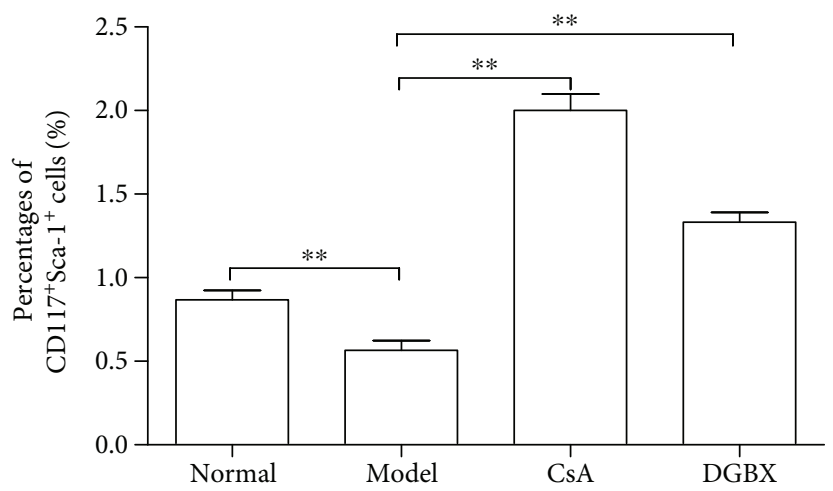

(b)

FIGURE 2: Effects of DGBX on proliferation of hematopoietic stem cells in bone marrow of mice with aplastic anemia (AA). (a) The percentage of CD117+Sca-1+ hematopoietic stem cells in the bone marrow cells of mice after 4 weeks of treatment. A, normal group, B, model group, C, group treated with cyclosporine A, and D, group treated with DGBX. (b) Results are presented in the bar charts. Data are presented as mean $\pm \mathrm{SD}, n=6 .{ }^{* *} P<0.01$.

2.9. Western Blot Analysis. BMCs $\left(1 \times 10^{7}\right)$ from each mouse were lysed in $0.5 \mathrm{~mL}$ of lysis buffer (Sigma, St. Louis, MO, USA). The extracts were cleared by centrifuging at $10,000 \mathrm{~g}$ and $4^{\circ} \mathrm{C}$ for $15 \mathrm{~min}$ and diluted with the lysis buffer to achieve about $2 \mathrm{mg} / \mathrm{mL}$ protein concentration. Protein samples were separated on $10 \%$ SDS-PAGE and transferred onto nitrocellulose membranes (Amersham Pharmacia Biotech, Uppsala, Sweden). The membranes were incubated with primary antibodies, including anti-T-bet/Tbx21antibody (1:1000), anti-Fas antibody $(1: 1000)$ (Abcam, Cambridge, MA, USA), and anti-mouse-caspase-3, anti-mouse-cleaved caspase-3 antibody, anti-mouse-eukaryotic initiation factor 2 (eIF2) $\alpha$ (D7D3), anti-mouse-phospho-eIF2 $\alpha$ (Ser51) (D9G8), anti-mouse-Fyn, anti-mouse interferon regulatory factor-1 (IRF-1), anti-mouse-signal transducer and activator of transcription (Stat) 1, and anti-mouse-Stat 3 rabbit monoclonal 

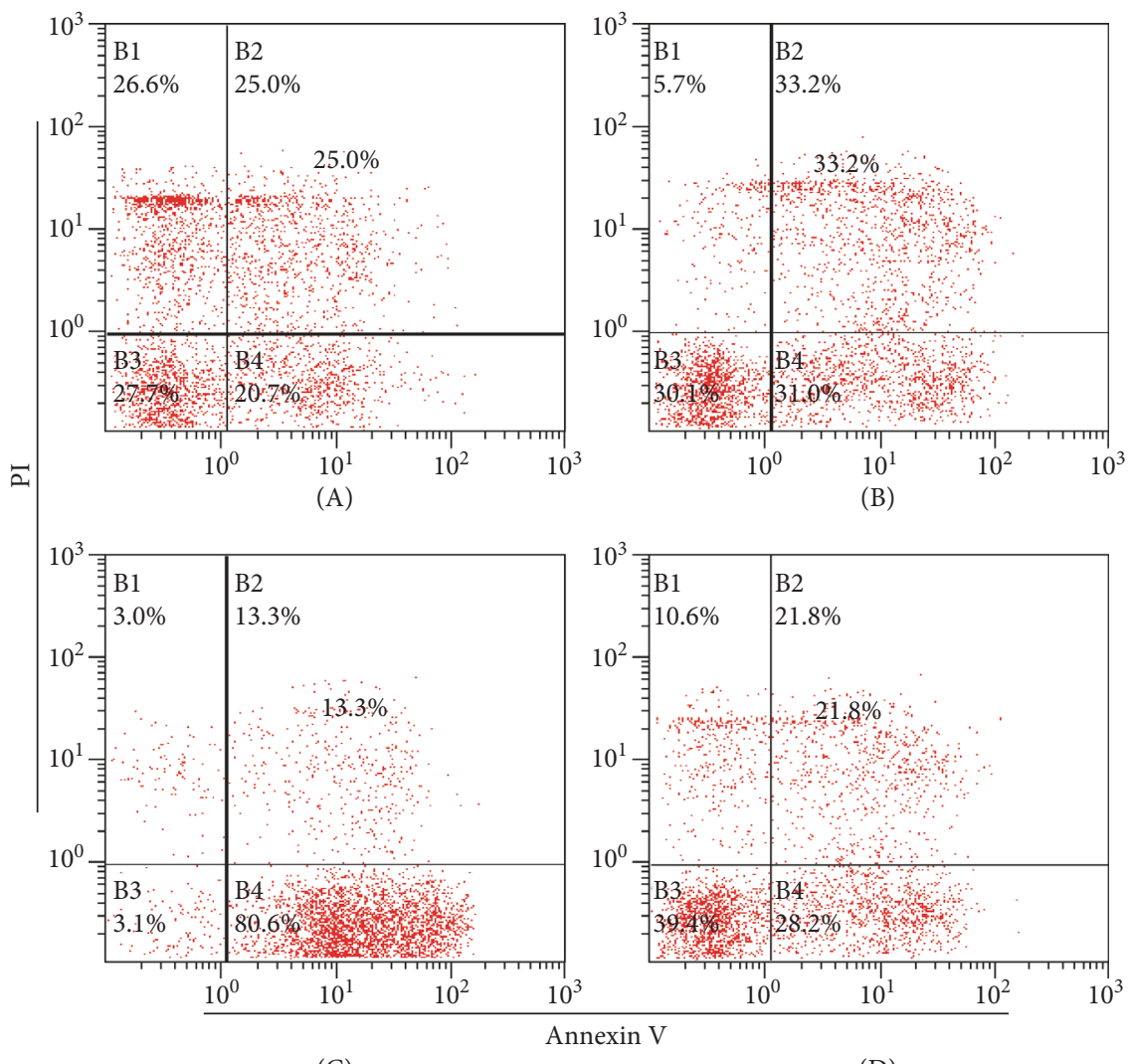

(C)

(D)

(a)

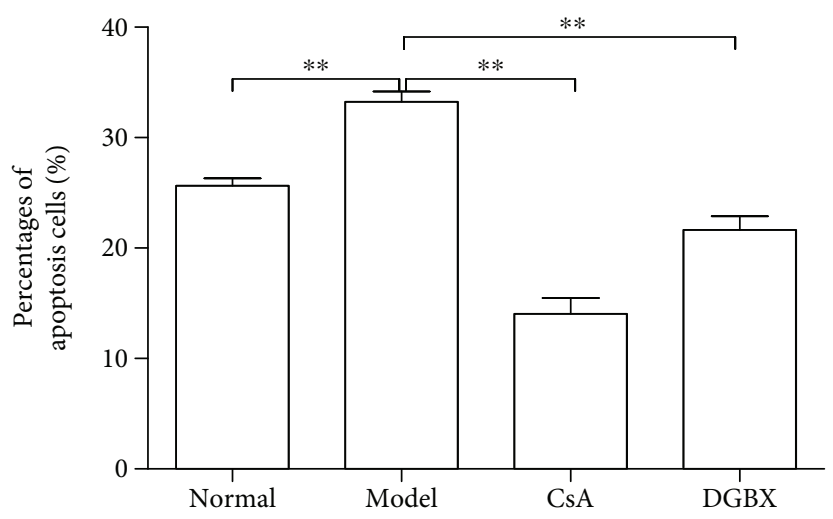

(b)

FIGURE 3: DGBX suppresses apoptosis of bone morrow cells in mice with aplastic anemia (AA). (a) The percentages of annexin $\mathrm{V}^{+} \mathrm{PI}^{+}$cells in bone marrow of mice after 4 weeks of treatment. A, normal group, B, model group, C, group treated with cyclosporin A, and D, group treated with DGBX. (b) Results are presented in the bar charts. Data are presented as mean $\pm \mathrm{SD}, n=6 .{ }^{* *} P<0.01$.

antibodies $(1: 1000$, CST, Boston, MA, USA) and incubated with horseradish peroxidase-conjugated secondary antibody (CST). All immunoreactive proteins were visualized with SuperSignal West Pico Chemiluminescent Substrate (Thermo Scientific, Rockford, IL, USA). Densitometry plots showing protein expression were normalized to GAPDH.

2.10. Statistical Analysis. All data were presented as mean \pm standard deviation (S.D.). The statistical analyses were performed using SPSS13.0 (SPSS Inc., Chicago, USA). One-way analyses of variance (ANOVA) followed by the TukeyKramer test for multiple comparisons were used to compare the treatment groups. A $P$ value of $<0.05$ was considered statistically significant.

\section{Results}

3.1. Characteristics of Pure Compounds from the Herbal Formula DGBX. In this study, high-resolution MS was performed in negative and positive ion modes to obtain 

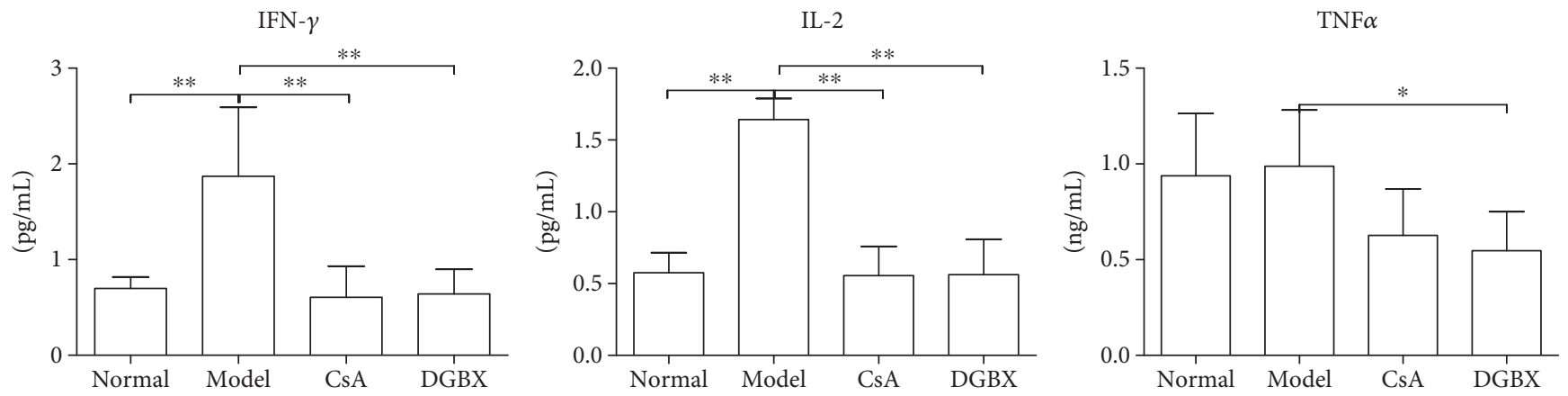

FIGURE 4: Evaluation of interferon $\gamma$ (IFN $\gamma$ ), interleukin-2 (IL-2), and tumor necrosis factor $\alpha$ (TNF $\alpha$ ) levels in sera of mice with aplastic anemia (AA) after treatment by using enzyme-linked immunosorbent assay. The results are presented in the bar chart. Data are presented as mean $\pm \mathrm{SD}, n=6 .{ }^{*} P<0.05,{ }^{* *} P<0.01$.

complete information about the chemical constitution of DGBX. The peak MS spectrum has been presented in Figure 1. Eighteen constituents were identified based on the accurate mass and relative ion abundance of the target peaks. The identified compounds are shown in Table 1.

3.2. Effects of DGBX on Proliferation and Apoptosis of HSCs in AA Mouse Model. Sca-1 and c-kit (CD117) are the major phenotypic markers for mouse HSPC subset [10]. Therefore, we quantified $\mathrm{CD} 117^{+} \mathrm{Sca}-1^{+}$cells to assess the percentages of HSCs in total BMCs. As shown in Figure 2, the amount of HSCs in model group significantly decreased compared with that in normal group $(P<0.01)$. HSC proliferation was significantly induced in DGBX group compared with that in the model group $(P<0.01)$.

We also observed the percentage of apoptosis in BMCs by AnnexinV-PI staining using a flow cytometer. The apoptosis ratio of BMCs in model group was significantly higher than that in normal group $(P<0.01)$. DGBX significantly inhibited the apoptosis of BMCs; apoptosis ratio in DGBX group significantly lower than that in model group $(P<0.01)$ (Figure 3).

3.3. Inhibition of IFN $\gamma, T N F \alpha$, and IL-2 Production in $A A$ Mice by DGBX. As shown in Figure 4, the levels of IFN $\gamma$ and IL-2 in model group were significantly higher than those in normal group $(P<0.01)$, and there was no significant difference in TNF $\alpha$ levels between the groups. After treatment with CsA and DGBX, the abnormally high levels of IFN $\gamma$ and IL-2 significantly decreased $(P<0.01)$ and the levels of TNF $\alpha$ also significantly decreased compared with that in the model group $(P<0.05)$. These results indicated that DGBX treatment could inhibit the production of inflammatory factors and that the inhibitory effect of DGBX was equivalent to that of CsA.

3.4. Effects of DGBX on Activation of SLAM/SAP Signaling Pathway. The SLAM-SAP signaling is an important pathway in T cell activation when engaged with ligands of T cell receptor [11]. SAP/SLAM recruits Fyn (a member of the Src family of kinases) that inhibits the expression of IFN $\gamma$ [12]. As shown in Figure 5, immunofluorescence analysis showed that the SLAM/SAP double-stained cells in the bone marrow of
AA mice significantly decreased compared with that in normal group $(P<0.01)$ and the decline was significantly inhibited by DGBX treatment $(P<0.01)$ (Figure 5$)$. However, DGBX treatment had no effect on the protein expression of Fyn.

3.5. DGBX Interferes with T-bet Expression and Distribution in AA Mice. T-bet, a transcription factor, binds to the IFN $\gamma$ promoter region and induces gene expression [13]. As shown in Figure 6, the T-bet ${ }^{+}$-stained cells significantly increased in bone marrow of AA mice $(P<0.01)$ and T-bet expression significantly increased $(P<0.01)$ compared with that in normal group. DGBX treatment significantly inhibited the levels of $\mathrm{T}^{-}$bet $^{+}$cells and protein expression. Furthermore, the regulatory effect of DGBX was equivalent to CsA.

3.6. Effects of DGBX on Activation of Eukaryotic Initiation Factor $2 \alpha$ in BMCs of AA Mice. Phosphorylation of the $\alpha$-subunit of eIF2 is a well-documented mechanism of downregulation of protein synthesis under various stress conditions. Western blot analysis demonstrated that eIF $2 \alpha$ expression in BMCs was not different between the groups. The phosphorylation level of total eIF2 $\alpha$ in DGBX group showed a decreasing trend compared with that in the model group $(P<0.05)$ (Figure 7$)$.

3.7. DGBX Regulates Expression of Key Molecules of FasMediated Apoptosis Signaling Pathway in BMCs of $A A$ Mice. As shown in Figure 8, the levels of Fas, caspase-3, and cleave caspase- 3 in model group were significantly higher than those in normal group $(P<0.01)$. DGBX significantly downregulated the expression of caspase- 3 and cleaved caspase- 3 compared with that in the model group $(P<0.05$ or $P<0.01)$. Interestingly, the regulatory effects of DGBX were superior to those of CsA. However, DGBX did not exert a regulatory effect on the expression of Fas in BMCs of AA mice.

3.8. DGBX Regulates Expression of Interferon Regulatory Factor 1 and Signal Transducer and Activator of Transcription 1 and 3 (Stat1 and Stat3) in BMC of $A A$ Mice. IRFs play an important role in the defense against pathogens, autoimmunity, lymphocyte development, cell growth, and susceptibility to transformation. Some effects of IFN $\gamma$ are mediated through IRF-1, which inhibits the transcription of 


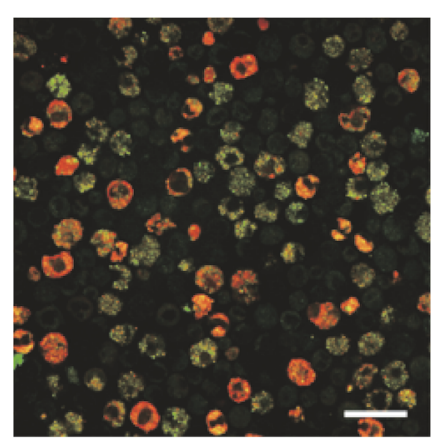

(A)

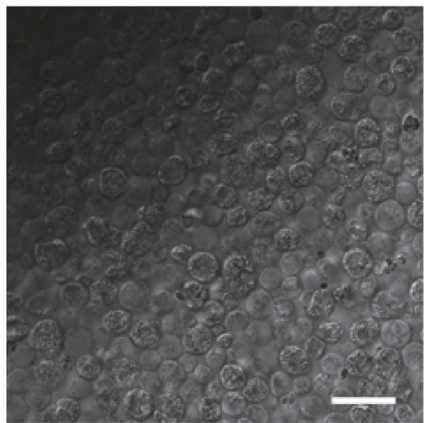

(E)

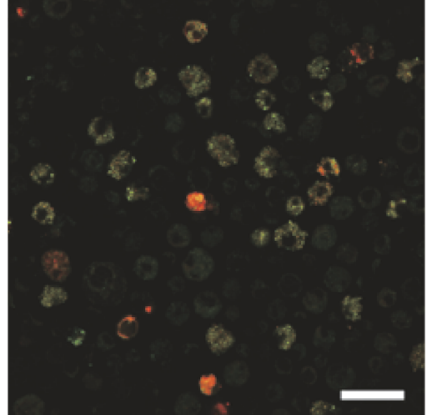

(B)

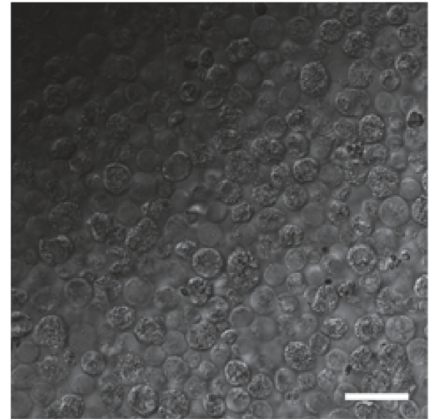

(F)

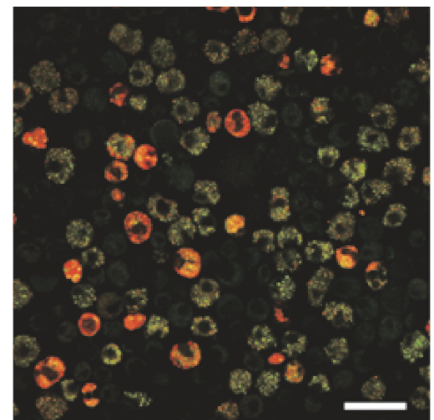

(C)

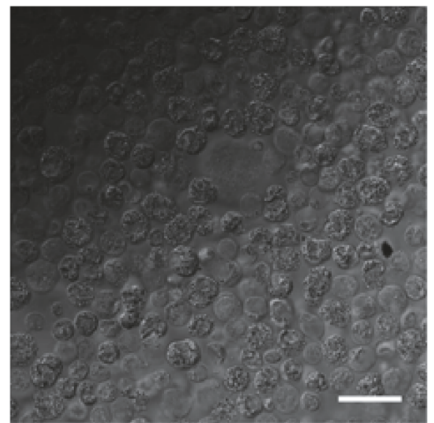

(G)

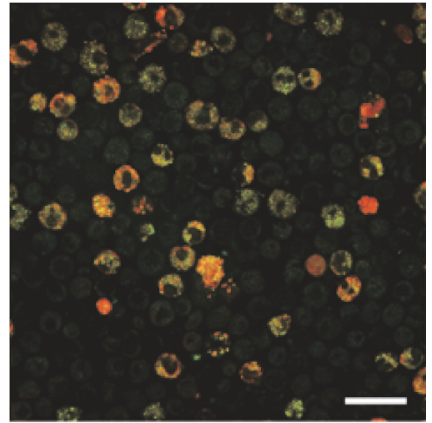

(D)

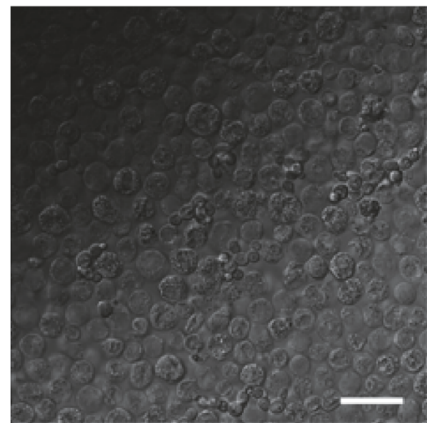

(H)

(a)

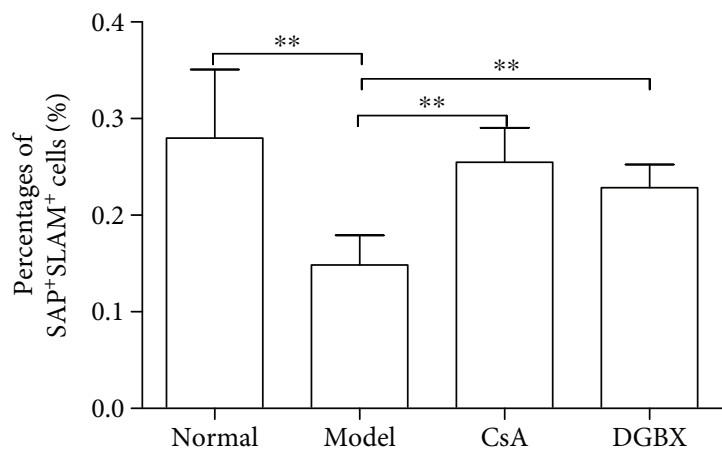

(b)

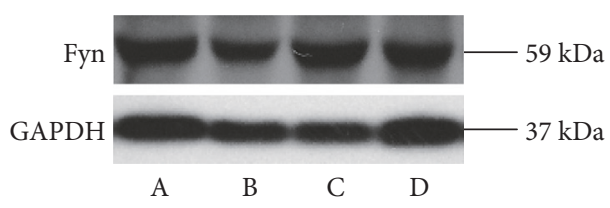

(c)

Fyn

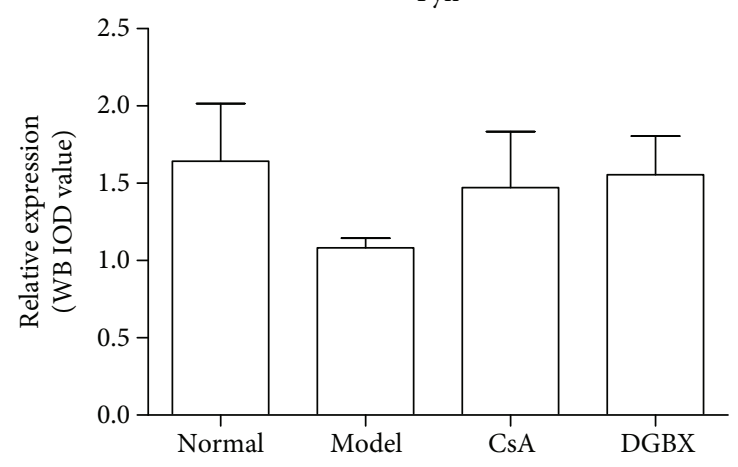

(d)

FIGURE 5: DGBX attenuates interferon $\gamma$ (IFN $\gamma$ ) expression by regulating the activation of SLAM/SAP signal. (a) BMCs were stained for CD150/SLAM (red) and SH2D1A/SAP (green) antibodies and observed by confocal immunofluorescence microscopy. The bottom pictures were obtained in bright field. The double-stained cells (yellow) were quantified with ImageJ. (b) Results are presented in the bar charts. The scale bar corresponds to $100 \mu \mathrm{m}$ throughout. A, normal group, B, model group, C, group treated with cyclosporin A, and D, group treated with DGBX. (c) Fyn was assessed in whole BMC lysates by Western blot analysis. (d) The results are presented in the bar chart. GAPDH was used as an internal control. Data are presented as mean $\pm \mathrm{SD}, n=6 .{ }^{* *} P<0.01$. 


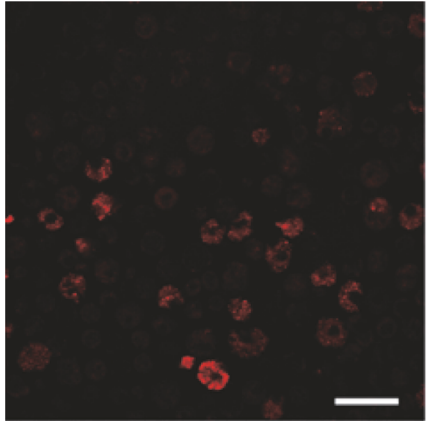

(A)

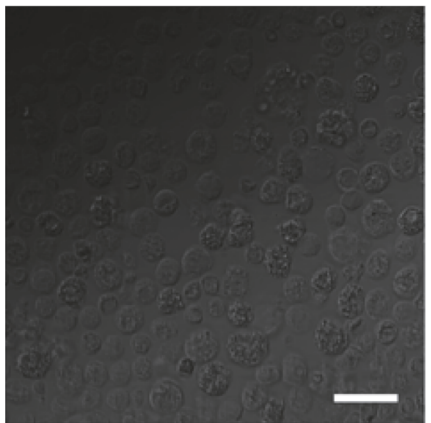

(E)

(b)

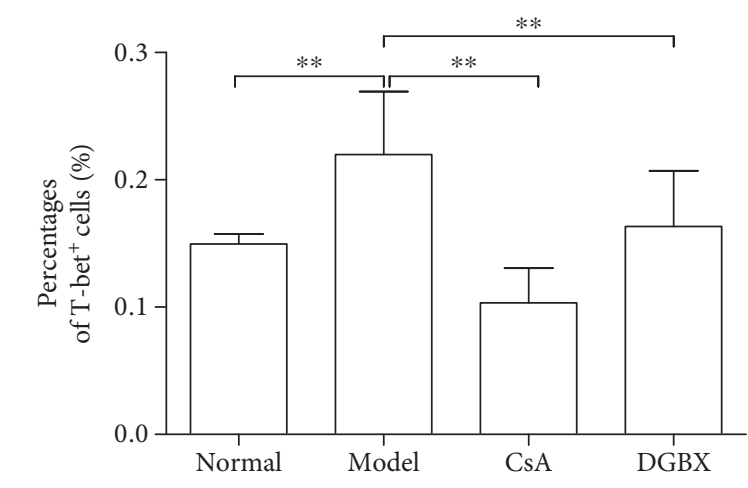

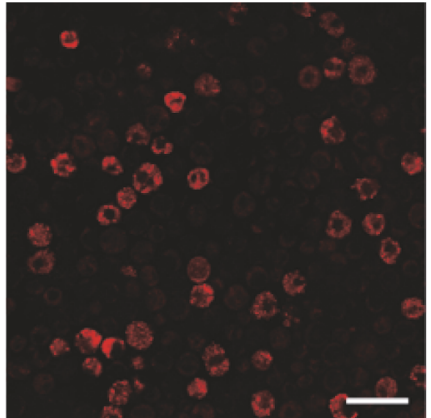

(B)

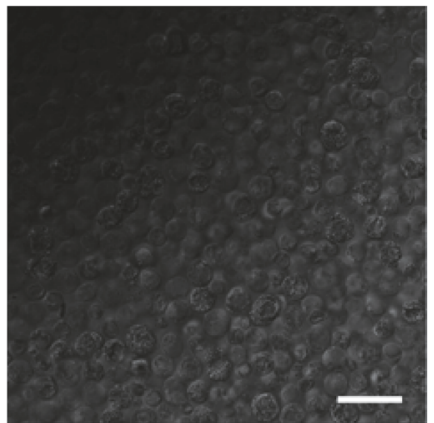

(F)

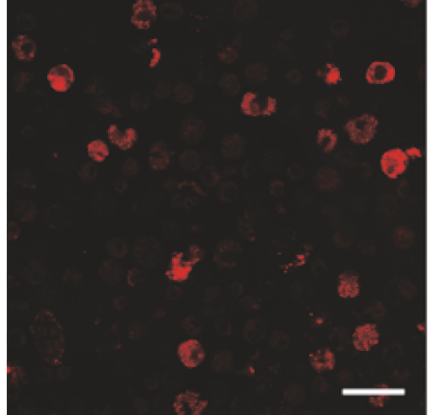

(C)

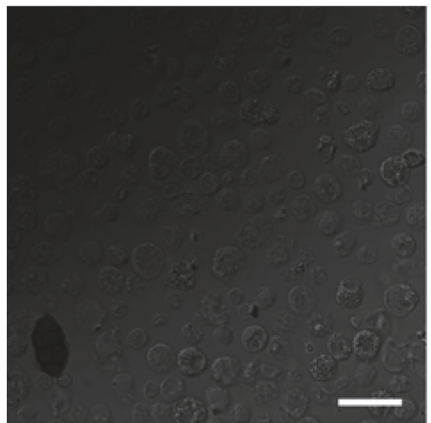

(G)

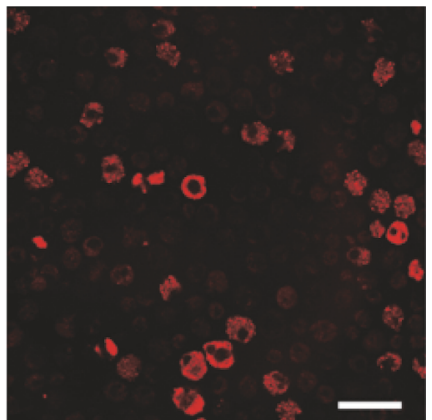

(D)

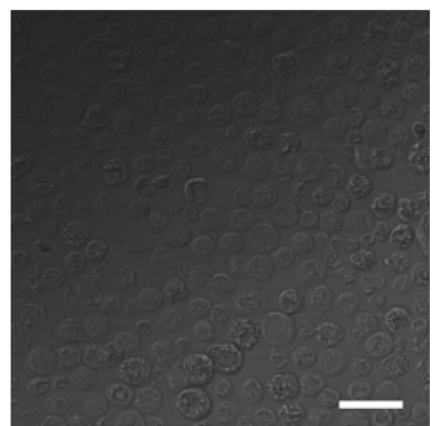

(H)

(a)

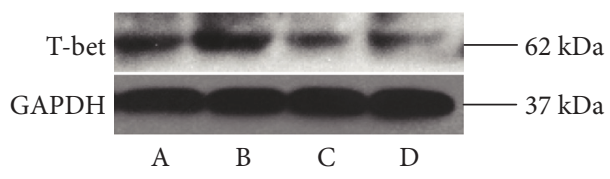

(c)

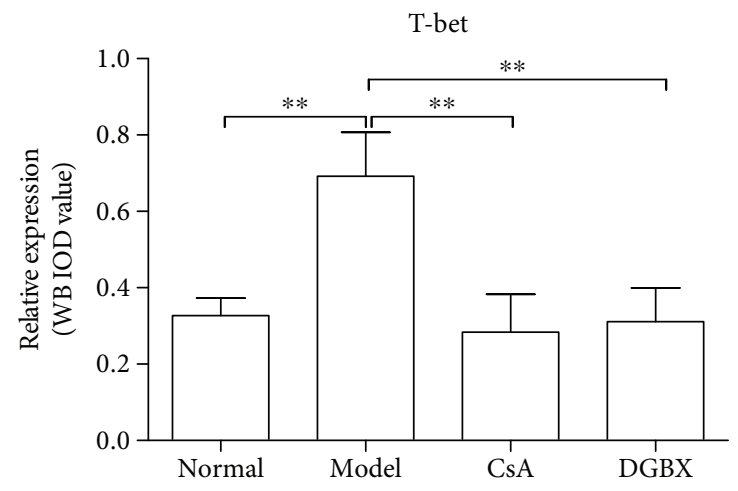

(d)

Figure 6: Expression and distribution of T-bet in bone marrow cells (BMCs) of mice with aplastic anemia (AA) after treatment. (a) Staining for T-bet (red) in BMCs was observed by confocal immunofluorescence microscopy. The bottom pictures were obtained in bright field. The stained cells (red) were quantitated with ImageJ. (b) Results are presented in the bar charts. The scale bar corresponds to $100 \mu \mathrm{m}$ throughout. A, normal group, B, model group, C, group treated with cyclosporin A, and D, group treated with DGBX. (c) The expression of T-bet was evaluated in whole BMC lysates by Western blot analysis. (d) The results are presented in the bar chart. Data are presented as mean \pm SD, $n=6 .{ }^{* *} P<0.01$. 


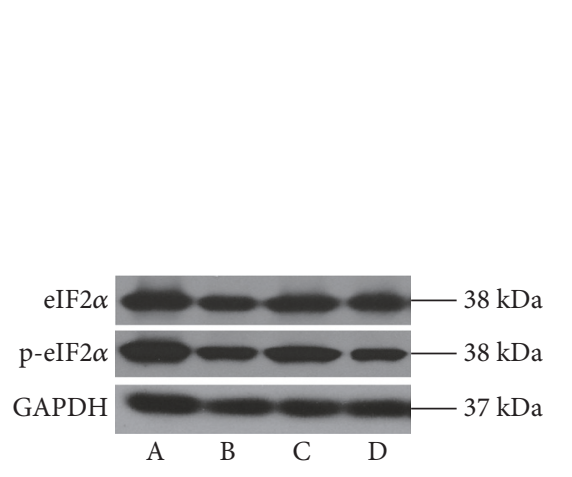

(a)

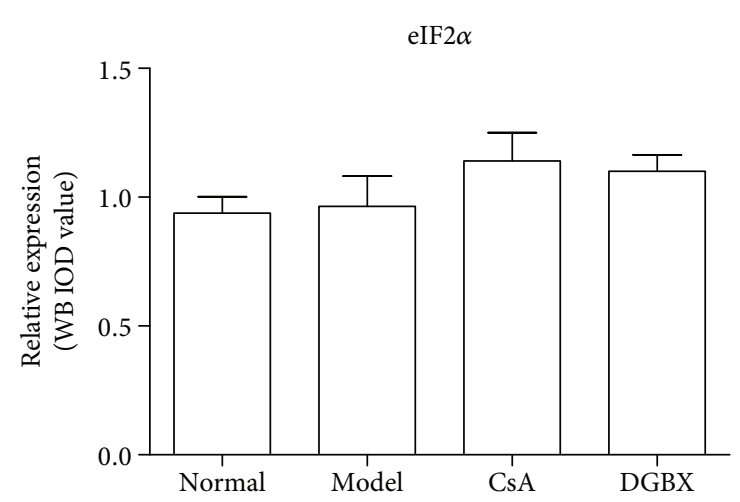

(b)

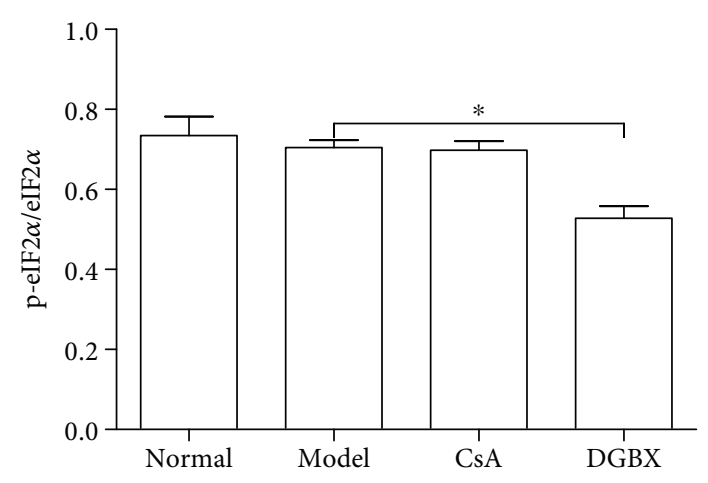

(c)

FIGURE 7: Effects of DGBX on phosphorylation of eIF2 $\alpha$ in bone marrow cells (BMCs) of mice with aplastic anemia (AA). (a) The expressive levels of eIF $2 \alpha$ and phospho-eIF $2 \alpha$ were estimated in whole BMC lysates by Western blot analysis. (b) Results are presented in the bar charts. A, normal group, B, model group, C, group treated with cylosporin A, and D, group treated with DGBX. (c) The ratio of phospho-eIF2 $\alpha$ and total eIF2 $\alpha$ is presented in the bar chart. GAPDH was used as the internal control. Data are presented as mean $\pm \mathrm{SD}, n=6 .{ }^{*} P<0.05$.

cellular genes and entry into the cell cycle. Stat 1 and Stat 3 contribute to this function [12]. The protein levels of Stat1 in the model group were significantly higher than those in AA mice $(P<0.01)$. DGBX downregulated the abnormally high expression of Stat1 $(P<0.05)$, but had no regulatory effect on the expression of IRF-1 (Figure 9).

\section{Discussion}

In TCM, combinatory therapeutic strategies based on patient symptoms and characteristics are often adopted to treat several diseases [14]. The Chinese herb formulae consist of several types of medicinal herbs or minerals, and multiple components could act on multiple targets and exert synergistic therapeutic efficacies [15]. Recently, LC-MS becomes an essential tool for analyzing the compounds of the herbal constituents in TCM complex formulae [16]. Eighteen constituents were identified by high-performance liquid chromatography-electrospray ionization/mass spectrometry from freeze-dried boiled aqueous extract of DGBX. The major components of DGBX-lyophilized powder were identified to be magnoflorine (3), tetradehydroscoulerine (7), jatrorrhizine (9), ononin (10), epiberberine (11), coptisine (12), palmatine (15), berberine (16), and calycosin (17) by Peak View Software analysis. Magnoflorine exhibits immunomodulatory effects on neutrophil and $\mathrm{T}$ cell-mediated immunity [17]. Coptisine can inhibit IL- $1 \beta$-induced inflammatory response by suppressing the nuclear factor-kappa $B$ (NF- $\kappa \mathrm{B})$ signaling pathway [18]. Jatrorrhizine plays a critical cell-protective role in $\mathrm{H}_{2} \mathrm{O}_{2}$-induced cell apoptosis owing to its antioxidative property [19]. Palmatine plays an important role in osteoclast apoptosis by regulating the inducible nitric monoxide synthase (iNOS) system [20]. Berberine can also decrease cell apoptosis induced by LPS by suppressing iNOS protein expression [21]. Calycosin reduces oxidative stress by regulating the activation of the PI3K/Akt/GSK- $3 \beta$ signaling pathway and suppressing osteoclastogenesis through inhibition of MAPK and NF- $\kappa \mathrm{B}$ activation [22]. AA is an acquired bone marrow failure syndrome resulting from immunemediated destruction of HSC. Therefore, we suggest that the functions of these herbal constituents of DGBX contribute to interaction with multiple targets and exert synergistic therapeutic efficacy in immunosuppression and hematopoiesis against AA.

Dysregulation in HSC cycling contributes to AA by enhancing differentiation over self-renewal or inducing apoptosis of HSCs [23]. Acquired AA exhibits increased levels of circulating IFN $\gamma$. IFNs have the pathogenic functions of impairing the proliferation of primitive HSPCs [24] and inducing apoptosis [25]. These evidences support that IFNs can impair hematopoiesis by attenuating HSC function. Antigen-presenting cells present antigens to $\mathrm{T}$ lymphocytes 


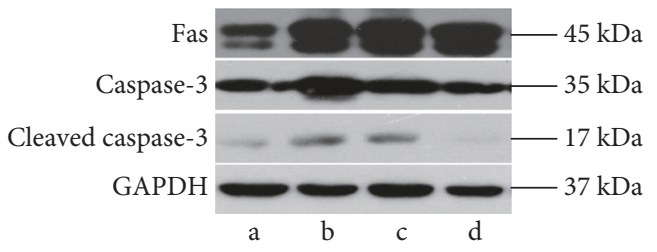

Caspase-3

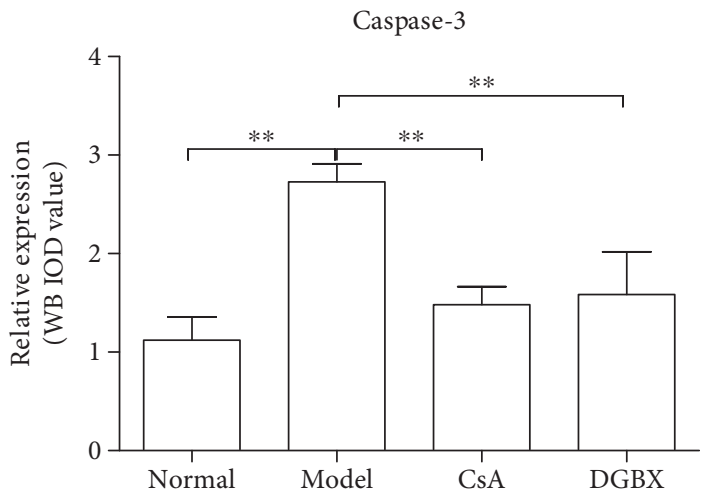

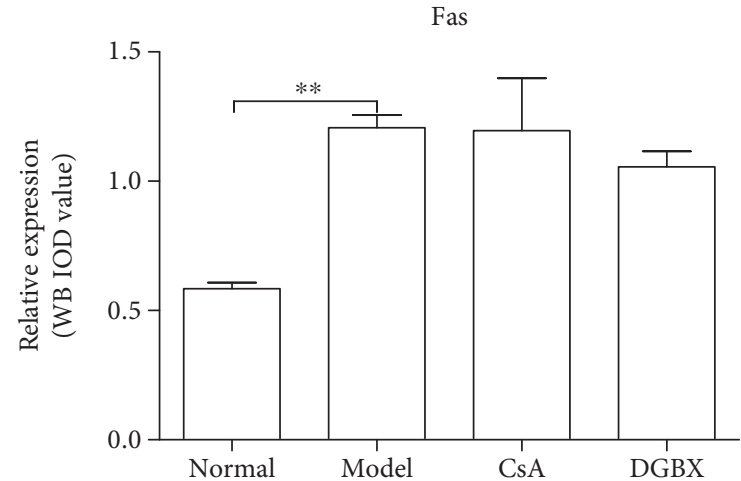

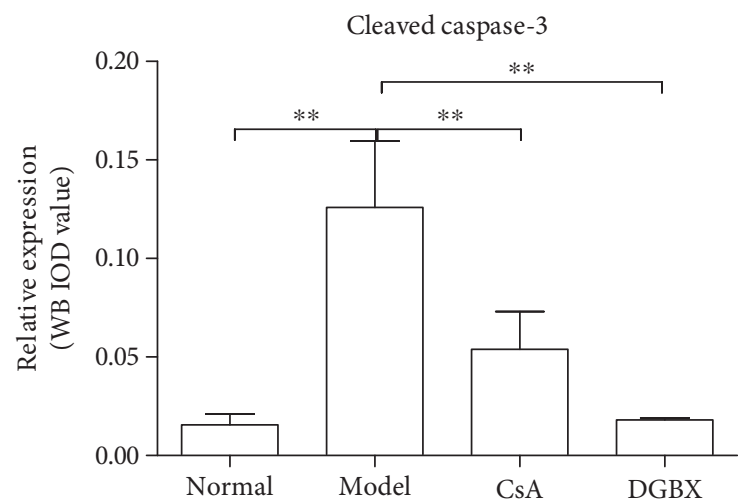

FIGURE 8: Effect of DGBX on the expressions of key molecule of Fas-dependent apoptosis pathway in AA mice. The protein levels of Fas, caspase-3, and cleave caspase-3 were estimated in whole BMC lysates by Western blot analysis. Results are presented in the bar charts. Data are presented as means $\pm \mathrm{SD}, n=6 .{ }^{* *} P<0.01$.

and trigger T cells to proliferate. The prototypical Th1 transcription factor T-bet binds to the IFN $\gamma$ gene promoter region and induces gene expression [26]. CD150/SLAM is a prototypical member of glycoprotein receptors on hematopoietic cells. It is unique in its binding to the signaling molecule SAP [27]. The binding of SAP and Fyn modulates the SLAM function in IFN $\gamma$ expression and then decreases IFN $\gamma$ gene transcription [12]. Constitutive T-bet expression and low SAP levels are observed in AA. Activated T cells also produce proinflammatory cytokine at abnormal levels. Increased production of IL-2 leads to polyclonal expansion of T cells. These immune responses initiate the immune-mediated $\mathrm{T}$ cell destruction of BMCs. We observed that the levels of circulating IFN $\gamma, \mathrm{TNF} \alpha$, and IL-2 in AA mice decreased to levels similar to those of normal group after treatment with DGBX. The quantity of SLAM/SAP double-stained cells significantly decreased, and the expression of Fyn was suppressed in BMCs by DGBX treatment. In addition, DGBX could inhibit the expression of T-bet, decreasing the level of T-bet-positive cells in the bone marrow. We believe that the immunosuppressive mechanisms of DGBX include SLAMmediated T cell proliferation and IFN $\gamma$ production for attenuating the immune-mediated destruction of bone marrow.

In the pathogenesis of the immune destruction of hematopoiesis, aberrant immune response induced IFN $\gamma$ production as well as TNF $\alpha$ and IL-2. IFN $\gamma$ and TNF $\alpha$ activate the $\mathrm{T}$ cell cellular receptors and Fas receptor [28]. Trimerization of Fas receptor interacts with Fas-associated death domain, activates caspase-3, and ultimately promotes HSC apoptosis [29]. IFN $\gamma$ can also activate the cell signaling cascade of IRF-1, contributing to the inhibition of the transcription of cellular genes and induction of eIF-2 phosphorylation for suppressing protein synthesis. IFN $\gamma$ induces IRF-1 binding to IFN-stimulated response elements, subsequently activating Stat 1 and Stat3, inducing gene transcription associated with inflammation, and contributing to the immunemediated destruction of the bone marrow [30]. This pathophysiology results in hematopoietic failure in AA. In this study, we found that the expression of phosphor-eIF $2 \alpha$, Stat1, Stat 3 , and caspase-3 significantly decreased in AA mice treated with DGBX. There were no regulatory effects of DGBX on the expression of Fas and IRF-1. These results indicated that DGBX could inhibit the aberrant immune response and the apoptosis of HSCs by modulating the activation of Stat/JAK/IRF-1 pathway and Fasdependent pathway.

CsA is a calcineurin inhibitor. It has selective effect on T cell functions by direct inhibition of the expression of nuclear regulatory proteins, contributing to reduction of $\mathrm{T}$ cell proliferation and activation. Severe AA can respond to CsA alone. Allogeneic bone transplantation and immunosuppressive treatment with antithymocyte globulin and CsA can significantly increase the 10-year survival rate in patients with severe AA [31]. In this study, we also assessed the significant 

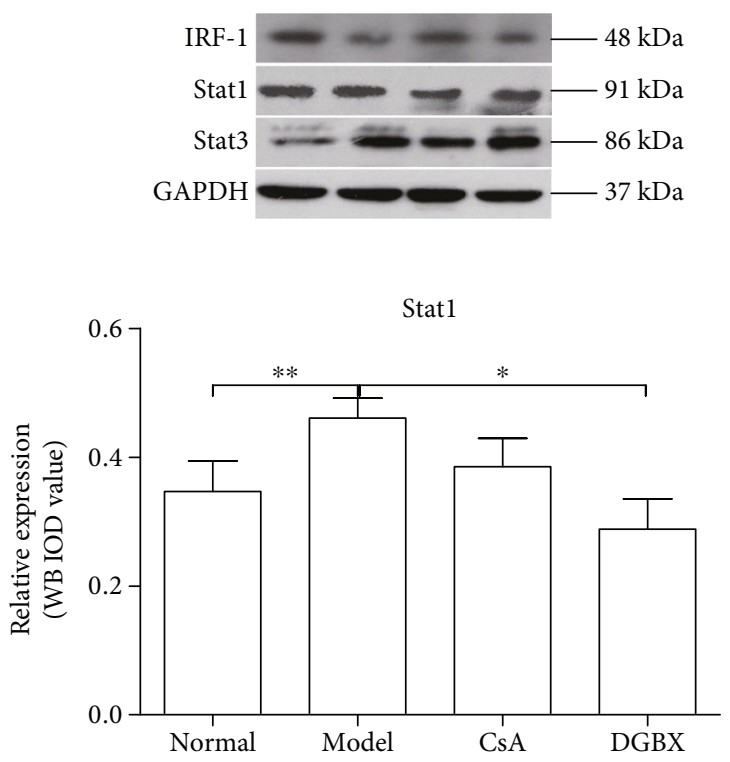

IRF-1
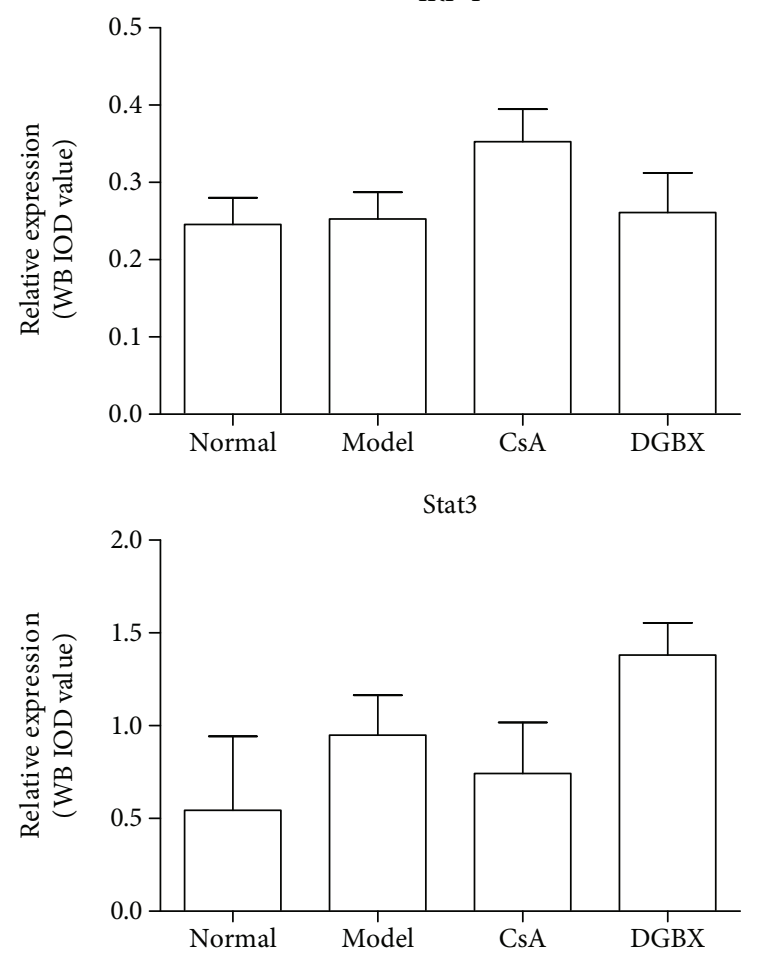

FIGURE 9: Effects of DGBX on regulation of expression of key molecules of interferon regulatory factor-1-mediated JAK/Stat signaling pathway in mice with aplastic anemia (AA). IRF-1, Stat1, and Stat3 expressions were estimated in whole BMC lysates by Western blot analysis. Data are presented as mean $\pm \mathrm{SD}, n=6 .{ }^{*} P<0.05,{ }^{* *} P<0.01$.

effects of CsA on the inhibition of proinflammatory cytokine and related protein expression, which participate in the immune response. CsA has many side effects, such as kidney damage and hepatotoxicity. These side effects can be managed by dose reduction [12]. Multiherbal formulas based on traditional medicine have been scientifically verified for use in complementary and alternative therapy for various diseases. Formulae composed of a mixture of natural products can target multiple sites. In our study, DGBX was verified as an effective medicine for AA treatment. It can also avoid kidney damage, hepatotoxicity, and other adverse reactions of drugs including CsA. Our results indicated that the immunosuppressive effects that aided recovery of the hematopoietic function of HSCs in hematogenesis in AA mice were equivalent or exceeded those of CsA.

\section{Conclusions}

Our data indicated that DGBX could attenuate IFN $\gamma$ production by interfering in SLAM/SAP signaling and production and distribution of T-bet in T cells, exert immunosuppressive effects by modulating the activation of the Stat/JAK/IRF-1 pathway, restrain cell apoptosis by intervening in Fasdependent pathway, and eventually attenuate immunemediated destruction of HSCs, repair hematopoietic failure, and recover the hematopoietic function of HSCs in hematogenesis for AA therapy. DGBX can be used in traditional medicine against AA as a complementary and alternative immunosuppressive therapeutic formula.

\section{Conflicts of Interest}

The authors declare that there is no potential conflict of interest.

\section{Authors' Contributions}

Jingwei Zhou, Xue Li, Peiying Deng, and Yi Wei contributed equally to this work.

\section{Acknowledgments}

This work was supported by the National Natural Science Foundation of China (no. 81373583).

\section{References}

[1] A. M. Risitano, "Immunosuppressive therapies in the management of immune-mediated marrow failures in adults: where we stand and where we are going," British Journal of Haematology, vol. 152, no. 2, pp. 127-140, 2011.

[2] M. M. Horowitz, "Current status of allogeneic bone marrow transplantation in acquired aplastic anemia," Seminars in Hematology, vol. 37, no. 1, pp. 30-42, 2000.

[3] S. Rosenfeld, D. Follmann, O. Nunez, and N. S. Young, "Antithymocyte globulin and cyclosporine for severe aplastic anemia: association between hematologic response and longterm outcome," Journal of the American Medical Association, vol. 289, no. 9, pp. 1130-1135, 2003. 
[4] W. L. Wang, S. Y. Sheu, Y. S. Chen et al., "Enhanced bone tissue regeneration by porous gelatin composites loaded with the Chinese herbal decoction Danggui Buxue Tang," PLoS One, vol. 10, no. 6, article e0131999, 2015.

[5] M. Yang, G. C. Chan, R. Deng et al., "An herbal decoction of Radix astragali and Radix angelicae sinensis promotes hematopoiesis and thrombopoiesis," Journal of Ethnopharmacology, vol. 124, no. 1, pp. 87-97, 2009.

[6] Y. Liu, H. G. Zhang, and X. H. Li, "A Chinese herbal decoction, Danggui Buxue Tang, improves chronic fatigue syndrome induced by food restriction and forced swimming in rats," Phytotherapy Research: PTR, vol. 25, no. 12, pp. 1825-1832, 2011.

[7] X. Yang, C. G. Huang, S. Y. Du et al., "Effect of Danggui Buxue Tang on immune-mediated aplastic anemia bone marrow proliferation mice," Phytomedicine: International Journal of Phytotherapy and Phytopharmacology, vol. 21, no. 5, pp. 640-646, 2014.

[8] J. Wang, T. Zhang, L. Zhu, C. Ma, and S. Wang, "Anti-ulcerogenic effect of Zuojin pill against ethanol-induced acute gastric lesion in animal models," Journal of Ethnopharmacology, vol. 173, pp. 459-467, 2015.

[9] J. Li, H. Chen, Y. B. Lv et al., "Intraperitoneal injection of multiplacentas pooled cells treatment on a mouse model with aplastic anemia," Stem Cells International, vol. 2016, Article ID 3279793, 6 pages, 2016.

[10] S. E. Vazquez, M. A. Inlay, and T. Serwold, “CD201 and CD27 identify hematopoietic stem and progenitor cells across multiple murine strains independently of kit and Sca-1," Experimental Hematology, vol. 43, no. 7, pp. 578-585, 2015.

[11] H. C. Chuang, J. D. Lay, W. C. Hsieh et al., "Epstein-Barr virus LMP1 inhibits the expression of SAP gene and upregulates Th1 cytokines in the pathogenesis of hemophagocytic syndrome," Blood, vol. 106, no. 9, pp. 3090-3096, 2005.

[12] N. S. Young, "Current concepts in the pathophysiology and treatment of aplastic anemia," Hematology/the Education Program of the American Society of Hematology American Society of Hematology Education Program, vol. 2013, pp. 76-81, 2013.

[13] C. L. Williams, M. M. Schilling, S. H. Cho et al., "STAT4 and T-bet are required for the plasticity of IFN-gamma expression across Th2 ontogeny and influence changes in Ifng promoter DNA methylation," Journal of Immunology, vol. 191, no. 2, pp. 678-687, 2013.

[14] T. Liu, H. Cao, Y. Ji et al., "Interaction of dendritic cells and T lymphocytes for the therapeutic effect of Dangguiliuhuang decoction to autoimmune diabetes," Scientific Reports, vol. 5, article 13982, 2015.

[15] L. Wang, G. B. Zhou, P. Liu et al., "Dissection of mechanisms of Chinese medicinal formula Realgar-Indigo naturalis as an effective treatment for promyelocytic leukemia," Proceedings of the National Academy of Sciences of the United States of America, vol. 105, no. 12, pp. 4826-4831, 2008.

[16] Q. Chen, S. Xiao, Z. Li, N. Ai, and X. Fan, "Chemical and metabolic profiling of Si-Ni decoction analogous formulae by high performance liquid chromatography-mass spectrometry," Scientific Reports, vol. 5, article 11638, 2015.

[17] W. Ahmad, I. Jantan, E. Kumolosasi, and S. N. Bukhari, "Standardized extract of Tinospora crispa stimulates innate and adaptive immune responses in Balb/c mice," Food \& Function, vol. 7, no. 3, pp. 1380-1389, 2016.
[18] K. Zhou, L. Hu, W. Liao, D. Yin, and F. Rui, "Coptisine prevented IL-beta-induced expression of inflammatory mediators in chondrocytes," Inflammation, vol. 39, no. 4, pp. 1558-1565, 2016.

[19] T. Luo, X. Shen, S. Li, T. Ouyang, and H. Wang, "The protective effect of jatrorrhizine against oxidative stress in primary rat cortical neurons," CNS \& Neurological Disorders Drug Targets, vol. 15, 2016.

[20] S. Ishikawa, M. Tamaki, Y. Ogawa et al., "Inductive effect of palmatine on apoptosis in RAW 264.7 cells," Evidence-Based Complementary and Alternative Medicine: eCAM, vol. 2016, Article ID 7262054, 9 pages, 2016.

[21] J. S. Shin, H. E. Choi, S. Seo, J. H. Choi, N. I. Baek, and K. T. Lee, "Berberine decreased inducible nitric oxide synthase mRNA stability through negative regulation of human antigen $\mathrm{R}$ in lipopolysaccharide-induced macrophages," The Journal of Pharmacology and Experimental Therapeutics, vol. 358, no. 1, pp. 3-13, 2016.

[22] G. H. Quan, H. Wang, J. Cao et al., "Calycosin suppresses RANKL-mediated osteoclastogenesis through inhibition of MAPKs and NF-kappaB," International Journal of Molecular Sciences, vol. 16, no. 12, pp. 29496-29507, 2015.

[23] J. N. Smith, V. S. Kanwar, and K. C. MacNamara, "Hematopoietic stem cell regulation by type I and II interferons in the pathogenesis of acquired aplastic anemia," Frontiers in Immunology, vol. 7, p. 330, 2016.

[24] A. M. de Bruin, O. Demirel, B. Hooibrink, C. H. Brandts, and M. A. Nolte, "Interferon-gamma impairs proliferation of hematopoietic stem cells in mice," Blood, vol. 121, no. 18, pp. 3578-3585, 2013.

[25] E. M. Pietras, R. Lakshminarasimhan, J. M. Techner et al., "Reentry into quiescence protects hematopoietic stem cells from the killing effect of chronic exposure to type I interferons," The Journal of Experimental Medicine, vol. 211, no. 2, pp. 245-262, 2014.

[26] E. E. Solomou, K. Keyvanfar, and N. S. Young, “T-bet, a Th1 transcription factor, is up-regulated in $\mathrm{T}$ cells from patients with aplastic anemia," Blood, vol. 107, no. 10, pp. 3983-3991, 2006.

[27] M. Morra, J. Lu, F. Poy et al., "Structural basis for the interaction of the free SH2 domain EAT-2 with SLAM receptors in hematopoietic cells," The EMBO Journal, vol. 20, no. 21, pp. 5840-5852, 2001.

[28] J. Maciejewski, C. Selleri, S. Anderson, and N. S. Young, "Fas antigen expression on CD34+ human marrow cells is induced by interferon gamma and tumor necrosis factor alpha and potentiates cytokine-mediated hematopoietic suppression in vitro," Blood, vol. 85, no. 11, pp. 3183-3190, 1995.

[29] G. Kroemer, L. Galluzzi, and C. Brenner, "Mitochondrial membrane permeabilization in cell death," Physiological Reviews, vol. 87, no. 1, pp. 99-163, 2007.

[30] M. S. Dicay, C. L. Hirota, N. J. Ronaghan et al., "Interferongamma suppresses intestinal epithelial aquaporin-1 expression via Janus kinase and STAT3 activation," PLoS One, vol. 10, no. 3, article e0118713, 2015.

[31] M. Fuhrer, U. Rampf, I. Baumann et al., "Immunosuppressive therapy for aplastic anemia in children: a more severe disease predicts better survival," Blood, vol. 106, no. 6, pp. 21022104, 2005. 


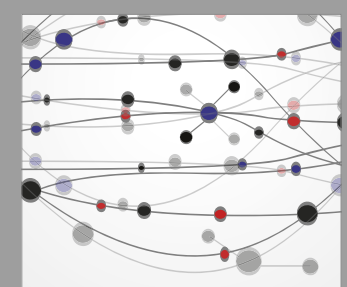

The Scientific World Journal
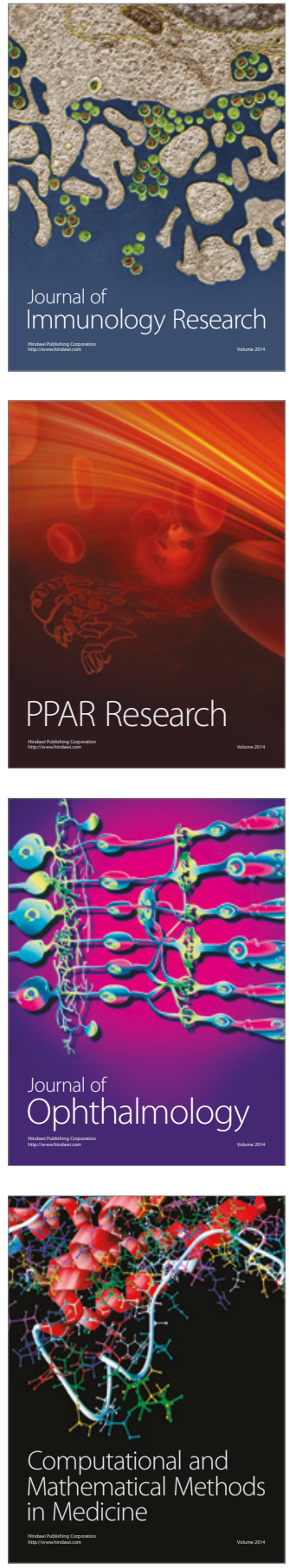

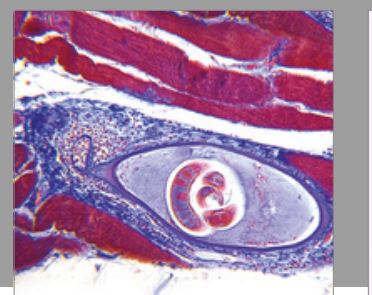

Gastroenterology Research and Practice
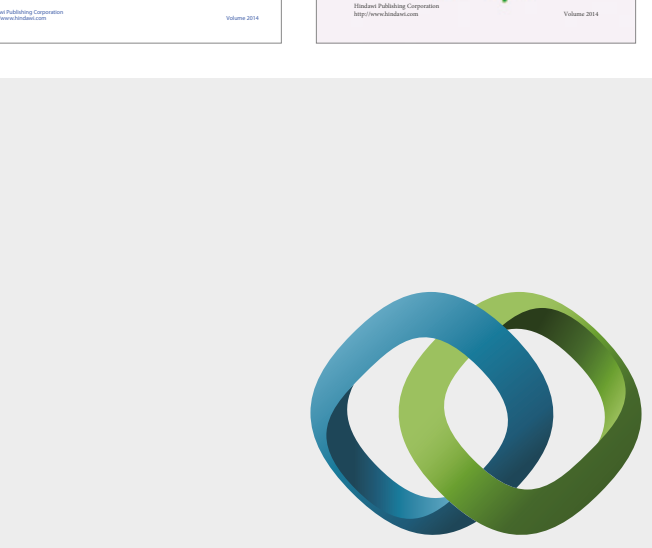

\section{Hindawi}

Submit your manuscripts at

https://www.hindawi.com
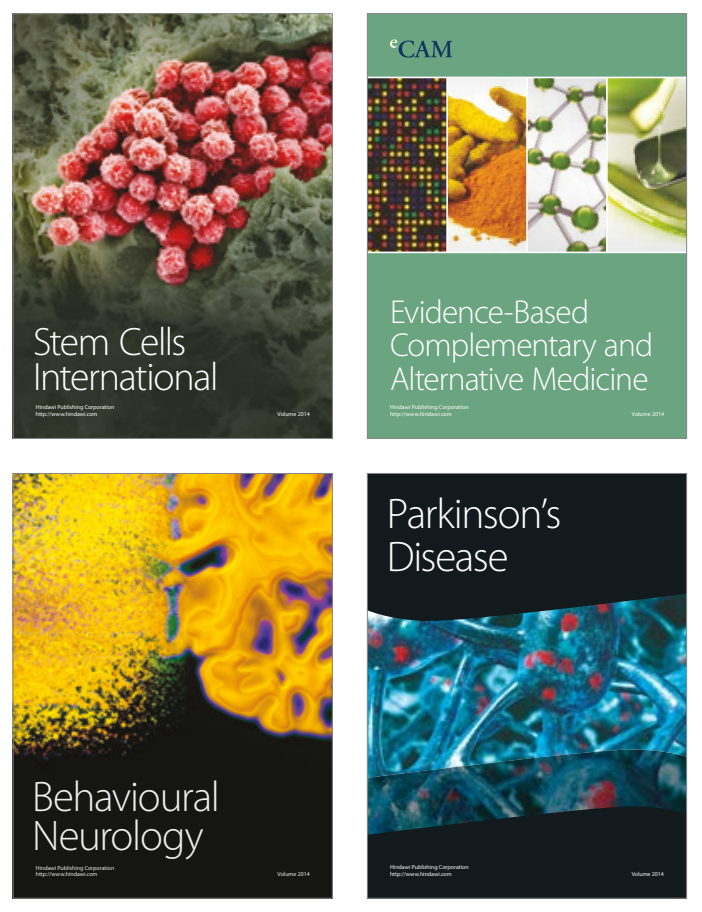
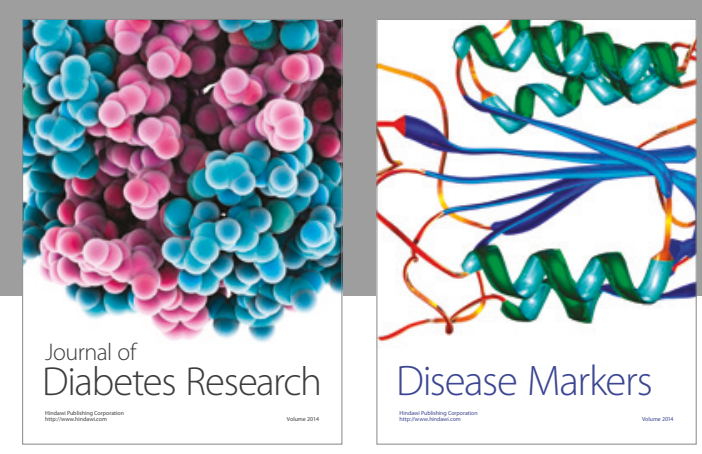

Disease Markers
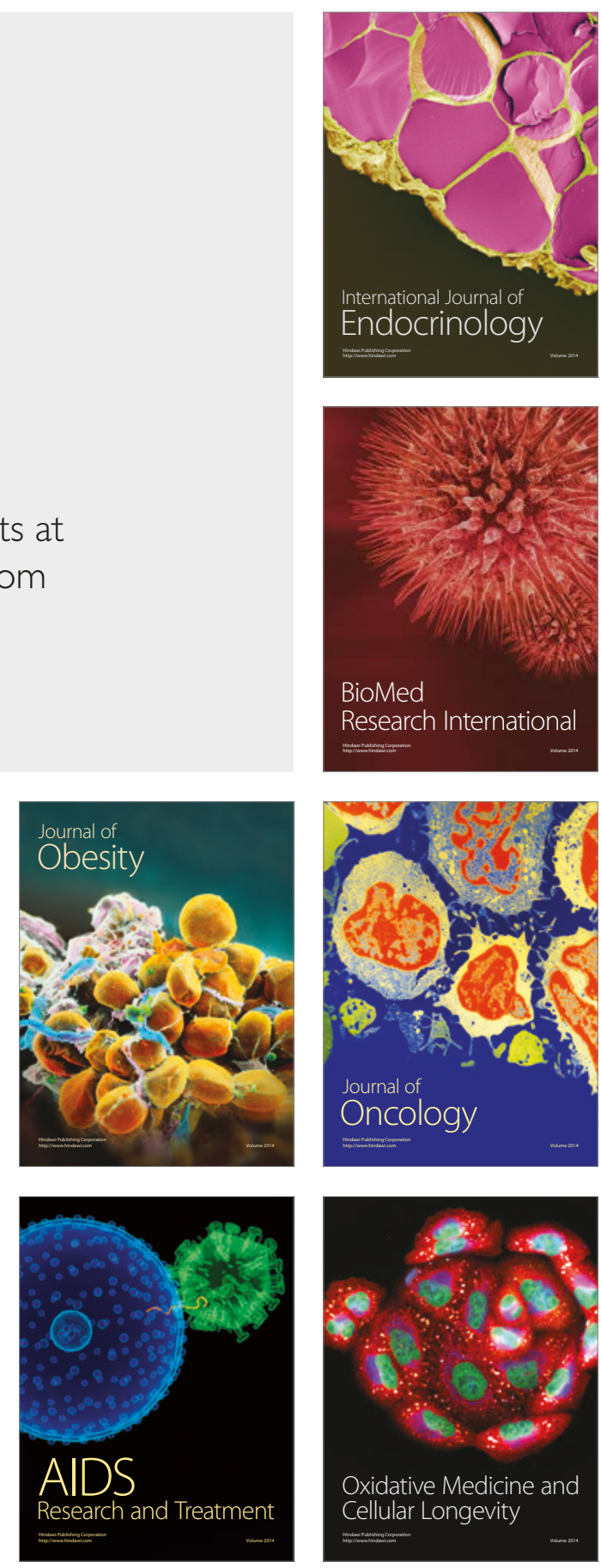